\section{Herramientas para la búsqueda de la equidad y la justicia social para todos}

\author{
George A. O. Alleyne
}

"La búsqueda de la equidad debe comenzar con una demostración de las desigualdades existentes, y esto solo se puede lograr si se dispone de información."
Tenemos que ser campeones de la búsqueda de esa equidad y justicia social que se traslucen en el concepto de Salud para todos.

Estas fueron mis palabras el día que asumí el cargo de Director de la Oficina Sanitaria Panamericana en 1995. Desde entonces, he tenido tiempo y ocasión para reflexionar acerca de lo que significa esa equidad para nosotros aquí en la Oficina y para los países de las Américas. La justicia social y la igualdad de todos los seres humanos no son conceptos novedosos; están incorporados en las constituciones de los países y a lo largo de los siglos los filósofos han señalado la necesidad de adoptar diversas medidas para garantizar que haya igualdad de oportunidades para todos.

Ha habido reiteradas expresiones de la preocupación por las brechas que existen entre ricos y pobres y por la consiguiente disparidad en su estado de salud. La búsqueda de la equidad debe comenzar con una demostración de las desigualdades existentes, y esto solo se puede lograr si se dispone de información. Sin dejar de reconocer la importancia que tiene la mala salud de las personas que se ven desfavorecidas por razones económicas o de otra índole, tenemos que darles alguna prioridad a las brechas o diferencias y a la posibilidad de reducirlas mediante intervenciones que ya han sido formuladas y probadas.

Con esta finalidad, la Organización Panamericana de la Salud (OPS) ha pasado los últimos ocho años tratando de definir un conjunto de datos centrales que puedan usarse para efectuar análisis de equidad, salud y evaluación de tendencias. Se ha hecho un esfuerzo especial por elegir indicadores básicos desagregados de acuerdo con algunos de los principales rasgos que se relacionan con la inequidad, tales como sexo, origen étnico, clase social, raza y distribución geográfica. Para la OPS, la búsqueda de una manera de garantizar la equidad en la provisión de esas medidas sanitarias y sociales que conducen a un buen estado de salud $\mathrm{y}$ en el acceso a ellas representa uno de los retos más importantes de brindar cooperación técnica como parte de su cometido. No es posible programar e implantar medidas eficaces destinadas a las poblaciones en mayor riesgo sin que primero se identifiquen las brechas en materia de salud y en la presencia de sus factores determinantes. 
"No es posible programar e implantar medidas eficaces destinadas a las poblaciones en mayor riesgo sin que primero se identifiquen las brechas en materia de salud y en la presencia de sus factores determinantes."

Este número especial de la Revista Panamericana de Salud Pública, que está dedicado al tema de la medición de las inequidades en el ámbito de la salud, representa un paso importante hacia lograr que los trabajadores de la salud pública tengan mayor acceso a la cooperación técnica para la medición de las desigualdades. Una de las principales contribuciones de este número de la Revista es la aplicación de metodologías epidemiológicas compatibles con los datos generados mediante los sistemas de información corrientes en los niveles nacional y local.

El presente número de la Revista es el resultado de una intensa colaboración entre el Programa Especial para el Análisis de la Situación de Salud, de la OPS, y el Centro Nacional de Epidemiología, bajo la Fundación Nacional de Salud del Ministerio de Salud del Brasil, cuyo objetivo ha sido poner herramientas metodológicas para la medición de las desigualdades en el campo de la salud al alcance de los profesionales de nivel local y nacional interesados en fortalecer su capacidad para analizar la situación de equidad a su alrededor y en enfocar adecuadamente sus intervenciones. Esta serie de artículos, al resaltar las bases metodológicas de la medición de las desigualdades en el terreno, ofrece una perspectiva global del uso de la epidemiología para investigar la equidad en materia de salud. Quisiera alentar a las autoridades sanitarias nacionales y locales, a los formuladotes de políticas, a los investigadores, a los profesionales de la salud y a toda persona que tenga interés en promover la equidad en materia de salud y en fomentar el progreso de la salud pública en nuestro continente a aprovechar este recurso invalorable. 